\title{
Algunos aspectos de las Cardiopatias y la mecánica circulatoria durante el embarazo
}

\author{
Por el doctor GUIILFRMO LOPEZ ESCOBAR \\ Jefe de Clinica Ginecologica de la Universidad Nacional
}

\section{INTRODUCCION}

Ha sido dicho con razón que: "La Maternidad es prerrogativa y privilegio de toda mujer, privilegio que nadie tiene el derecho de rehusar sin una razón poderosísima". Por esto nos alegramos de que hayan sido abandonados los viejos aforismos de Peters (57) para la mujer cardíaca: "Doncella no te cases, esposa no concibas, madre no amamantes".

Las opiniones son hoy más optimistas. Ya desde hace treinta años Osler (54) escribia: "Las mujeres por lo general soportan las lesiones valvulares mejor que los hombres, en parte porque llevan una vida más sosegada, en parte porque las coronarias son menos afectadas y en parte por la mayor frecuencia de lesiones mitrales. Embarazo y parto son factores agravantes en ellas, pero menos serios de lo que ciertos autores nos querrían hacer creer". Durante las últimas décadas, un mejor control de las cardíacas embarazadas y un mejor entendimiento de la mecánica circulatoria han traído como resultado una conducta y terapéutica mejores. Esto ha disminuido considerablemente el porcentaje de una de las causas más frecuentes de mortalidad materna. Sin embargo un $25 \%$ de las muertes maternas son debidas a la presencia de una complicación cardíaca (37) y en un pasado reciente Fraser (30) afirmaba que entre las muertes maternas no causadas por complicaciones primariamente obstétricas, las cardíacas solamente cedían el primer lugar a las enfermedades pulmonares no tuberculosas.

$\mathrm{Y}$ es más: muchos conceptos sobre cardíacas embarazadas, conceptos en que la divergencia de opiniones es tan grande que, obviamente, la conclusión final no será alcanzada sino a través de mayores búsquedas e investigaciones (37). Fue pues con el objeto de adquirir una mejor comprensión del problema y de contribuír en algo a su solución que el presente trabajo se inició.

\section{CONSIDERACIONES GENERALES SOBRE LAS CARDIOPATIAS DURANTE EL EMBARAZO}

\section{Diagnóstico:}

En la mayoría de los casos la paciente es vista por el obstetra cuando ya está embarazada. Para este entonces la influencia fisiológica del embarazo sobre la circulación se ha hecho ya manifiesta y el diagnóstico de enfermedad cardíaca es frecuentemente difícil (71). 
Cammenfolt (32) encontró que durante los meses finales del embarazo, un $16,3 \%$ de las embarazadas presentaban signos que sugerían enfermedad cardiaca sin que ésta pudiera demostrarse una vez pasada la gestación. Por lo tanto cada sintoma debe ser evaluado a la luz del estado gravidico. Cambios en signos auscultatorios son muy frecuentes sobre todo acentuación o desdoblamiento de los ruidos pulmonares (12). Soplos funcionales se presentan en un 5 a un $75 \%$ de todas las embarazadas (2-23-24-43). Ligera taquicardia, estrasístoles irregulares, disnea y edema en varios grados, son en circunstancias normales, un hecho común durante la gravidez y de todos es conocida la vieja controversia sobre dilatación o hipertrofia del músculo cardíaco durante el embarazo normal. El confundir un "corazón gestatorio" con un caso de enfermedad cardíaca llevaria a gastos, angustias y tratamientos innecesarios.

Así, pues, el diagnóstico debe ser basado sobre sintomas o signos en los que se pueda confiar. Hamilton (1) en su magnífico libro, trae los siguientes cuatro puntos, cada uno de los cuales él califica como de "sorprendentemente adecuados para seleccionar mujeres jóvenes cardíacas":

1) Definido aumento del tamaño del corazón, si es necesario confirmado por Rayos $\mathrm{X}$.

2) Un soplo diastólico.

3) Desórdenes peligrosos del ritmo.

4) Signos o historia de descompensación cardíaca.

\section{Incidencia:}

A través de la literatura (1-2-1923-29-34-33-43-49-52-53) la incidencia de enfermedad cardíaca orgánica en mujeres embarazadas varía de un $0,16 \%$ a un 4 o $5 \%$. Esta discrepancia es probablemente debida a diferencias en criterio diagnóstico y situación geográfica. Bien sabida es la desigual distribución del Reumatismo Cardíaco. En general la mayoría de las cifras caen entre un 1 o $2 \%$. Las estadísticas son aún más disparejas cuando se trata del número de muertes maternas ocasionadas por complicaciones cardíacas. Algunas investigaciones traen datos tan altos como $25 \%$ (38) mientras que otras presentan sólo un $0,7 \%$ (49). Estas variaciones son, talvez, el resultado de diferente tratamiento. Con los adelantos en el manejo de pacientes cardíacos la tasa de mortalidad ha disminuido definitivamente de lo que acostumbraba ser un 10 a $15 \%$ a un 3 o $4 \%$ (44-24).

En el período de ocho años de 1939 a 1946 inclusive, 16.385 mujeres dieron a luz en el Desloge y el St. Mary's, dos de los Hospitales del Grupo de la Universidad de St. Louis. En 68 de ellas se encontró una lesión cardiaca definitiva. Una incidencia de $0,41 \%$.

\section{Etiología:}

El Reumatismo Cardiaco Crónico hace las más de las enfermedades cardíacas durante el embarazo (3443-59-61). Esto es de esperar puesto que la edad en que la mujer concibe más frecuentemente, es entre los 20 y los 40 años. El Reumatismo Cardíaco Agudo es muy raro (40) 
aunque mucho más factible de ocurrir cuanto más joven sea el grupo de futuras madres.

El resto de los casos está constituído por: Enfermedad Cardíaca Congénita, Enfermedad Cardio-vascular Luética o Hipertensiva, Endocarditis Bacterial Subaguda y ocasionalmente Tirotoxicosis (25).

La Estenosis Mitral es la lesión más común en el reumatismo valvular crónico de las pacientes obstétricas, así como en las mujeres jóvenes no embarazadas. Hamilton (1) en sus casos halló un $83,3 \%$ de estenosis mitral con o sin dilatación valvular. El diagnóstico clínico de la lesión valvular es hecho mucho más fácilmente, claro está, cuando hay signos cardíacos, pero a pesar de esto, Sheehan y Sutherland (62) en un estudio de gran número de autopsias de embarazadas cardíacas, encontraron que el diagnóstico había sido hecho correctamente tan sólo en la mitad de los casos.

Los factores etiológicos encontrados en el grupo de pacientes de la Universidad de St. Louis, anteriormente mencionado fúeron: Reumatismo Cardíaco $85,3 \%$, Congénitas cardíacas $3,5 \%$, Enfermedad Cardio-vásculo-renal Hipertensiva, $5 \%$, Taquicardia paroxística, 5,5\%.

\section{Clasificación:}

Antes de la Primera Guerra Mundial, la clasificación de las enfermedades cardíacas se hacía principalmente desde un punto de vista anatómico (65). Más tarde los cardiólogos reconocieron que el estado funcional del músculo cardíaco y la reserva cardíaca eran un mejor índice. Pardee (55) en 1922 aplicó tal clasi- ficación a las cardíacas embarazadas y luégo bajo los auspicios de la New York. Heart Association fue introducida la clasificación funcional que ha sido tan ampliamente usada. Las pacientes son divididas de acuerdo con su función cardíaca, así:

Clase I: Pacientes cardiacas que son capaces de llevar a cabo ordinaria actividad física sin disconfort 0 dificultad alguna.

Clase II: Pacientes , cardíacas capaces de llevar a cabo ordinaria actividad física, pero que tienen dificultad al hacerlo.

Clase III: Pacientes cardiacas que tienen dificultad en llevar a cabo actividad física menos que ordinaria.

Clase IV: Pacientes cardiacas que son incapaces de llevar a cabo cualquier actividad física sin dificultad.

Posible Enfermedad Cardiaca: pacientes con signos o sintomas achácables al corazón pero en quienes el diagnóstic es incierto.

Enfermedad Cardíaca Potencial: Pacientes sin enfermedad cardíaca, pero a quienes es aconsejable vigilar por la historia de un factor etiológico que puede ser la causa de enfermedad cardíaca:

Las de la clase I es de esperar que no se descompensen durante el $\mathrm{cm}$ barazo. Las de la clase II probablemente no se descompensarán, pcro hay gran probabilidad de que las de la clase III lo hagan. Las de la clase IV son extremadamente difíciles de manejar y probablemente tendrán una alta mortalidad.

La aplicación de esta clasificación a la práctica es algunas veces muy difícil. Casos límites son comunes. Pacientes que en un principio son 
colocadas en la Clase I, con el progresivo aumento de trabajo del corazón debido al embarazo, tienen que ser cambiadas de clase. Debido a esto Hamilton (1) ha propuesto una clasificación diferente, con: un Primer Grupo que comprende todas las verdaderas cardíacas subdividiéndolas en Favorables y No Favorables, de acuerdo con etiología, factores anatómicos, etc. Un Segundo Grupo que correspondería a los casos llamados Posibles y Potenciales en la clasificación de la New York Heart Association y un Tercer Grupo formado por aquellas pacientes funcionales no verdaderas cardiacas.

Sin embargo en las manos de Jensen (2) y Opell (53) la clasificación de la New York Heart Association ha sido de gran valor y la reacción de las pacientes a las actividades diarias ha constituído un índice adecuado. Es necesario tener en cuenta sí, el hecho de que el estado cardíaco de una paciente durante el embarazo no es estático (65), sino que a más de la carga propia de la gravidez, hay factores extrínsecos que pueden precipitar la descompensación cardíaca. Hagamos una breve revista de éstos:

Factores superimpuestos que pueden precipitar la descompensación cardíaca:

Hay muchos factores adicionales que juegan un importante papel en el desencadenamiento de la descompensación. En general tales factores pueden ser hallados en un $50 \%$ de los casos (63). Algunos de ellos son extracardíacos y favorecerían la aparición de la descompensaçión en pacientes cuya patología cardíaca por sí sola pudiera no haber causado dificultad alguna. Otros son inherentes a la condición cardiaca misma. Rápidamente comentaremos los más importantes:

La edad tiene una importancia definitiva. La mayoría de las descompensaciones se producen en mujeres por encima de los 30 (27-53-63). Hamilton (1) halló duplicada la incidencia por encima de los 35. De ahí que sea aconsejable el que la cardíaca se case y dé a luz joven, aunque no debe olvidarse que son más frecuentes las reincidencias de la fiebre reumática aguda en las pacientes demasiado jóvenes.

A pesar de que el riesgo de descompensación se repite con cada embarazo sucesivo, la multiparidad no parece aumentar la frecuencia de ella si no es por el hecho de estar asociada con una más avanzada edad (1).

Infecciones intercurrentes, sobre todo del tracto respiratorio superior; anemia; obesidad; choques emocionales y sobre-ejercicio, que sean debidos a actividades sociales o a coito fatigantemente repetido, o a pesados trabajos caseros o a excursiones de compras a las que son tan aficionadas las mujeres; son todas cargas indeseables. No está por demás el recalcar sobre la necesidad de una vigilancia médica estricta.

La Fibrilación Auricular presente durante el embarazo, se acompaña de una gran mortalidad (43).

La Hipertrofia Cardíaca refleja un severo esfuerzo y sin embargo Sheehan y Sutherland (62) no encontraron en su estudio de autopsias 
indicacion que les permitiera suponer que descompensación y muerte eran mucho más frecuentes en pacientes con gran hipertrofia que en aquellas con poca.

Hipertensión, Endocarditis Bacterial, o el antecedente de una descompensación previa añaden, claro es, peligros peculiares no reflejados por la clasificación funcional pero que deben ser tenidos en cuenta.

\section{La sobrecarga del embarazo en} relación con la época más frecuente de descompensación:

El útero grávido con el feto y la placenta representa un órgano parásito de gran lecho vascular, por el cual existe un alto aumento de la circulación materna (4). Para ajustarse a tales cambios el organismo de la madre tiene que sufrir algunas alteraciones.

Durante el embarazo la frecuencia y la presión del pulso aumentan. El débito cardíaco aumenta en casi un 50\% (4-31-66). Hay también un aumento del volumen sanguíneo y del consumo de oxígeno (16). Estos son los cambios que constituyen la carga del embarazo sobre la circulación. Pero cuándo se halla esta carga en un máximum? Cuándo, por el hecho de que la carga gravídica ha alcanzado la cima, es la enferma cardíaca más propensa a descompensarse? La determinación de esțe máximum es de una gran importancia desde el punto de vista pronóstico y de la conducta con la cardíaca embarazada.

El trabajo era considerado como la época más peligrosa.
Desde que Burwell (10-11) ha demostrado la analogía entre la circulación placentaria y una fístula arterio-venosa, fuera lógico presumir que el tiempo de formación de la placenta, esto es hacia el tercero o cuarto mes, debería ser un tiempo peligroso. Los hechos clínicos no sวportan esta presunción.

Cohen y Thompson (16) han probado que el aumento del volumen sanguíneo y del débito cardíaco comienzan muy temprano en el embarazo y ascienden progresivamente para alcanzar una máxima altura alrededor del octavo o noveno mes lunar. Durante el último mes lunar el retorno hacia la normalidad principia. Hamilton (1) ha confirmado estos hechos clínicamente, sus casos presentaron una mayor incidencia de descompensación del octavo al noveno mes y es muy rara la aparición de ésta, por primera vez, durante el parto. La pequeña serie de casos presentados en este estudio parecen sustentar tales aserciones, cincuenta por ciento de los casos que presentaron signos de descompensación comenzaron a hacerlo del séptimo al octavo mes.

Una explicación adecuada del por qué de esta ligeración de la carga durante el último mes no ha sido encontrada. En varios estudios (2-5365) de casos numerosos no se ha hallado época especial del embarazo durante la cual fuera más probable que ocurriera la descompensación cardíaca. Esto, unido al hecho de que la mayoría de las muertes cardiacas ocurren después del parto (39) y de que nuestras observaciones (ver $\mathrm{Ca}$ pítulo III) en embarazadas normales muestran una disminución neta 
de la capacidad vital y ligero aumento de la velocidad de circulación en los dias inmediatamente postpartum, nos ha hecho pensar que talvez el período más peligroso sea en estos días inmediatamente después del parto.

Es fácil ver, en todo caso, que la última palabra no ha sido dicha $\mathrm{y}$ que se hacen necesarias más investigaciones acerca de la materia.

\section{Conducta y Pronóstico de la Embarazada Cardíaca:}

"No importa que tan altamente califique uno su habilidad para estimar la capacidad cardíaca de una paciente, ni siquiera se piense que uno pueda predecir con certeza cómo tal corazón individual reaccionará durante el embarazo". Hamilton (37). De ahi la falacia de interrumpir un embarazo solamente basados en el diagnóstico de una definida lesión cardíaca orgánica, puesto que al mayor número de las cardíacas les irá bien durante un embarazo llevado bajo apropiado cuidado. Daly (21) ha recalcado que casi toda mujer cardíaca puede llevar con éxito un embarazo.

Marx White (75) afirma: "Es mi creencia que cuanto más uno entiende del corazón enfermo, cuanto más uno ha tratado desórdenes funcionales y orgánicos de la circulación, tanto más profundamente uno siente la responsabilidad para con la madre y para con el hijo y tanto menos a menudo seria consideración se le es dada a la interrupción del embarazo antes de término".

En la mayoria de las clínicas en donde los esfuerzos del obstetra y del cardiólogo han sido combinados en el cuidado bien coordinado de la mujer embarazada cardíaca, el número de abortos terapéuticos se ha reducido considerablemente (25). La cuidadosa vigilancia del embarazo ha suplantado la interrupción del mismo. Jensen (2) opina que desde 1930 en el St. Louis Maternity Hospital, ningún embarazo ha sido interrumpido por enfermedad cardíaca hasta que el feto no fuera razonablemente viable y que no han habido razones para lamentar tal ${ }^{\circ}$ conservatismo.

Cuando una embarazada es vista por primera vez en descompensación cardiaca, las indicaciones para un aborto terapéutico son aún menores. Bajo ninguna circunstancia deben hacerse intentos de evacuar el útero, sea cual fuere el medio, mientras la paciente esté en descompensación congestiva (29). Esto es cierto tanto en los primeros como en los últimos meses.

"Descompensación cardíaca más parto forzadamente provocado es igual a muerte" (17). Es mucho más seguro conservar tal paciente en cama, bajo terapéutica médica, que someterla al esfuerzo adicional que un trabajo inducido representa. Si ella no sobrevive a las medidas médicas, es mucho menos probable que sobreviva al schock de un parto provocado (59).

Es de todos aceptado que el trabajo es, en general, bien soportado por la paciente no descompensada. Asi pues, ha sido sugerido que se induciera el trabajo antes de término con el objeto de tener con un feto más pequeño un trabajo más fácil. Esta solución aparentemente feliz no es correcta. Inducción hecha antes 
de término usualmente resulta, si se logra, en un tipo insatisfactorio de trabajo. Según Sampson y Rose un aumento del consumo de oxígeno sigue a la administración de Pituitrinas y aumento del consumo de oxígeno significa mayor trabajo cardíaco. Aún más, dos de las pacientes en el presente estudio, cayeron en descompensación por vez primera, después de repetida inducción médica. Todos estos hechos nos han permitido apreciar qué tan cierta es aquella frase de que "no hay medio mágico para hacer desaparecer el embarazo" (19), y de que la inducción en cardíacas debe ser usada, si lo es, con sumo cuidado.

\section{Descubrimiento precoz de la descomposición.}

Para establecer medidas terapeuticas adecuadas, la descompensación debe ser sospechada tan pronto como sea posible. El descubrimiento de la temprana descompensación está basado en las manifestaciones clínicas usuales, pero hay terreno para creer que la congestión pulmonar ocurre probablemente de manera más fulminante durante el embarazo que en otras épocas. Al decir de Opell (53) "sería precipitada por el embarazo".

Hamilton (1) cree que la presencia de estertores persistentes en la base de los pulmones es el que aparece más pronto de los signos de confianza.

Una marcada disminución de la capacidad vital, excepto inmediatamente después del parto, es también una evidencia temprana.
Edema, tos persistente y disnea progresiva serän de ayuda en el reconocimiento de la descompensación.

Presiones venosas altas no son un signo precoz, sino más bien, una indicación del grado de dificultad en las pacientes severamente descompensadas.

Las determinaciones de la Velocidad de Circulación, aunque a "grosso modo" un test útil, no parecen muy indicativas debido a la amplia escala de variaciones en los resultados.

Mendelson y Pardee (50) han mostrado que una taquicardia por encima de 110 por minuto con aumento del número de respiraciones por encima de 24 , durante el primer período de trabajo, precedió cada uno de sus casos de descompensación intra o post-partum, con tiempo suficiente como para estar sobre aviso de la inminente aparición de fenómenos congestivos.

Así, pues, el descubrimiento precoz de descompensación en la cardiaca embarazada debe estar basado, no en un síntoma, signo o test en particular sino en el conjunto de todos ellos y en la observación repetida y cuidadosa de la paciente.

\section{Medidas terapéuticas.}

La más simple y eficaz medida terapéutica en la embarazada cardíaca es el reposo en cama (14). Profilácticamente por una o dos semanas antes del parto es aconsejable a la mayoría de ellas. El reposo en cama debe ser, también, instituído en cualquier momento en que la des- 
compensacion cardiaca parezca más probable. Si hay signos reales de descompensación, el reposo absoluto, sin permitir que la paciente se levante para nada, debe ser recalcado. $Y$, una véz que la descompensación haya ocurrido, la enferma guardará cama hasta tres o cuatro semanas después del parto.

Durante la descompensación cardíaca hay mayores probabilidades de formación de trombos intracardía$\cos$ y son necesarias a lo menos tres semanas para que se adhieran firmemente a las paredes del corazón (1). Súmese a esto el conocido hecho de que los accidentes embólicos son más frecuentes en los días que siguen a procedimientos obstétricos $u$ operatorios.

La última de nuestras fatalidades cardíacas, no incluída en estas series, nos impresionó muchísimo. Se trataba de una multípara de edad, obesa, con embarazo avanzado, quien llegó al Servicio en severa descompensación. Fue atendida, tratada y dió a luz cuando aún estaba en ligera descompensación. Durante la primera semana post-partum, bajo cuidadosa terapia, mejoró notablemente. El edema y la dispnea desaparecieron. La capacidad vital aumentó de 1.000 a 1.450 centímetros cúbicos. La presión venosa descendió de 300 a 200 milímetros. La velocidad de circulación (brazo a lengua) que previamente era de 40 segundos disminuyó a 18 segundos. Ella se sentía mucho mejor y en general el pronóstico parecía ser bueno cuando repentinamente al octavo día del puerperio, después de haber sido colocada en el pato, la enferma se puso cianótica, extremadamente agita- da, dispneica, con movimientos convulsivos y finalmente cayó en estado semicomatoso para morir unas horas más tarde. El diagnóstico más probable fue embolía cerebral. Desgraciadamente la autopsia no fue permitida por los familiares.

Con la esperanza de disminuír accidentes como el descrito, hemos comenzado a usar profilácticamente el (3,3'-Metilenebis (4-Hidroxicumarina). Dicumarol durante las dos primeras semanas después del parto de cardiacas descompensadas, subrayando también la importancia de que durante este tiempo el reposo en cama sea absolutamente estricto. Nuestra experiencia a este respecto es aún corta y sólo el tiempo podrá decir si esta sugestión es de valor (*).

La reducción de fluídos, sedantes, dieta, diuréticos, Oxígeno y Digitalización son medidas bien, conocidas que no necesitan comentario.

(*) N. B.: Este trabajo fue presentado en abril de 1947. A fines del mismo año tuve oportunidad de asistir a las reuniones del "Staff" del Departamento de Ginecología del Columbia Medical Center en Nueva York y durante una de ellas, en un trabajo sobre este mismo tema presentado por uno de los miembros del Staff, con sorpresa y satisfacción oí presentar esta misma sugestión encomiásticamente, como uno de los probables medios para disminuír el porcentaje de muertes cardíacas maternas debidas a trombos y embolías. 


\section{Parto.}

La conducta en el parto mismo de las cardräcas es aún motivo de discusión, aunque la tendencia general hoy dia es hacia más partos por vía vaginal.

Después de toda intervención abdominal, la paciente debe pasar por un periodo de convalescencia a menudo más fatigoso que el trabajo mismo (22). Curtis y Mencielson (49) comunican 1.038 partos vaginales de cardíacas sin muerte alguna, mientras que en 45 casos por vía abdominal hubo tres muertes. En ambos grupos había cardíacas severas, clases III y IV.

Puesto que en general, morbilidar y mortalidad son mayores despucis de cesárea (34), la mayoría de los obstetras están de acuerdo en que la enfermedad cardíaca "per se" es rarísimamente, si acaso, una indicación para la operación cesárea (1-35).

La fuerza expulsiva del pujo cn el segundo período de trabajo que ocasiona un brusco aumento del consumo de oxígeno $(60)$ debe ser evitada con el uso de forceps y episiotomía.

La anestesia local (68) o en su defecto el éter dado con gran porcentaje de oxígeno es 'el anestésico más seguro en las embarazadas cardíacas.

Después del parto el uso de ur gran vendaje abdominal y almohadillas ha sido aconsejado (29-38). Esto tendería a contrarrestar la repentina disminución de presión intra-abdominal y la reducción de la circunferencia torácica, que son probablemente la causa de la disminución de la capacidad vital en esta época.
DURACION DEL TRABAJO Y TAMAÑO DEL RECIEN NACIDO EN MADRES CARDIACAS

Exisí la impresión general de que el trabajo en pacientes cardíacas es de más corta duración. A pesar de que pocas observaciones reales se han hecho, tal afirmación es oída de labios de muchos obstetras. Como posible explicación de esto, se ha sugerido que el tamaño de los recićn nacidos de madres cardíacas es menor que el promedio normal. Se ha dicho también que estos recién nacidos son más pequeños porque se han desarrollado en un ambiente un tanto anóxico. El feto creciendo en el útero de una cardíaca, especialmente si existe descompensación, recibiria sangre a baja tensión de oxígeno.

Los datos de las cardíacas del presente estudio no confirman tan generalizada creencia.

La duración del trabajo en setenta y seis partos de madres cardíacas, y el peso y medidas de los recién nacidos de ellas fueron tomados. El material fue obtenido de los siguientes hospitales: Firmin Desloge, St. Mary's, St. John's, St. Anthony's, y De Paul del Grupo de la Universidad de St. Louis.

En este grupo de pacientes había treinta primíparas, diez y siete multíparas de dos embarazos, diez de tres, cuatro de cuatro, cinco de cinco, cinco de seis, una de ocho y una de nueve embarazos previos. La duración del trabajo no fue tomada en once de ellas.

En el cálculo promedio del peso de los recién nacidos, los siguientes fueron excluídos por razones obvias: un par de gemelos $(2.100$ y 2.700 
gramos). Uno nacido muerto, macerado y probablemente post-maturo (4.180 gramos) y cinco prematuros.

El promedio de duración del trabajo en las cardiacas fue de: 14 horas $91 / 2$ minutos para las primíparas.

7 horas y 33 minutos para las multíparas.

Si correlacionamos estas cifras con el promedio dado por Fordel (28) para primípara y multipara: 14 horas y cinco minutos y 8 horas y cuarenta y un minuto respectivamente, la diferencia es nula.

Comparado con los promedios de Stander (3) 18 horas para primíparas y 12 horas para multíparas, nuestras cifras parecen un tánto menores. Esta disparidad es fácilmente cornprensible si consideramos que en las pacientes cardíacas la aplicación del forceps reemplazó usualmente el segundo período del trabajo.

El tamaño promedio de los recién nacidos de madres cardíacas fue:

Peso: 3135,5 gramos.

Longitud: $50,1 \mathrm{cms}$.

El promedio de longitud y peso de los recién nacidos de madres normales es dado generalmente (3) como de 50 centímetros y 3.200 gramos respectivamente. De nuevo aquí no se ve ninguna diferencia entre los normales y los nacidos de madres cardíacas.

Tampoco fueron apreciadas diferencias notables al comparar las medidas de las circunferencias cefálicas, torácicas o abdominales. (*).

(*) El Resumen de las Historias de las pacientes cardíacas fue omitida aquí.
DETERMINACIONES DE

CAPACIDAD VITAL $Y$

VELOCIDAD DE CIRCULACION

DURANTE EL EMBARAZO.

Mencionamos previamente que existen notables cambios en la mecánica de la circulación durante el embarazo, particularmente en las semanas que precedían y seguían al parto. Afirmamos también, que era en estas semanas cuando se presentaba una mayor incidencia de descompensaciones y fatalidades en las cardíacas.

Los tests más usados en Clínica General para estimar descompensación cardíaca son: Capacidad Vital, Presión Venosa y Velocidad de Circulación. Varios investigadores han estudiado las variaciones de estos tests durante el embarazo, pero sus resultados son bastante desacordes. la divergencia es aún mayon cuando se trata de los tres últimos meses del embarazo. Cualquiera que lea el capítulo sobre Capacidad Vital del Libro de Jensen (2) comprobará que no hay acuerdo alguno entre los resultados de los diversos autores. He creído de valor observar el comportamiento de la Capacidad Vital y la Velocidad de Circulación durante la época inmediatamente pre y post-partum.

Las observaciones fueron hechas sobre 55 embarazadas normales de la Clínica Obstétrica del Desloge Hospital. Veinticuatro de ellas eran multíparas y treinta y una primipara. Excepto dos que tenían una vaguísima historia de fiebre reumática durante la infancia, todas las demás eran normales desde el punto de vista historia y examen físico. 
Hubo también una que mas tarde desarrolló una ligera preeclampsia.

Las edades variaban de 15 a 43 años; la mayoría entre 20 y 30 .

Las determinaciones fueron hechas en la mañana, la mayor parte de las veces en ayunas o después de un muy escaso desayuno (una tostada).

Las siguientes instrucciones les eran repartidas cada vez que se las citaba para un test. Coma una ligera comida la noche anterior al día en que usted debe ir a la clínica. Vaya a cama temprano. No se bañe esa mañana. No se apresure al vestirse ni al coger'el bus o el tranvía ni al llegar al hospital.

Una vez en la clínica las pacientes permanecian una hora en reposo.

\section{Capacidad vital.}

El espirómetro de agua del "McKesson Metabolor" fue el empleado. A cada una de las pacientes se les enseñó cómo practicar el test y durante él continuamente se las instaba a dar un máximo rendimiento. La mayor de tres lecturas en decúbito dorsal y la mayor de tres lecturas en la posición sentada era anotada.

Un total de 1.260 oisservaciones fueron hechas, en las dos posiciones.

En 22 de las pacientes hubo determinaciones pre-natales solamente. En treinta $\mathrm{y}$ tres hubo determinaciones pre y post-natales. El número de lecturas pre-natales varió en cada paciente entre 2 y 6 y fueron hechas durante los últimos tres meses del embarazo. Las observaciones post-natales, en la mayoría de los casos fueron hechas el primer día, el séptimo día y cinco a seis semanas después del parto.
De las 55 pacientes originales, 4 fueron desechadas más tarde por falta de cooperación.

\section{Resultados.}

Como en la mujer no embarazada, las observaciones hechas en la paciente sentada son usualmente más altas que cuando ella está acostada. Así también, las mismas pacientes mostraron de semana en semana, fluctuaciones de 100 centímetros cúbicos promedio, por encima o debajo de las lecturas anteriores.

El factor entrenamiento fue importantemente notorio. En 59 por 100 de las pacientes, las lecturas hechas durante la primera visita fueron las más bajas del período prenatal.

Las cifras encontradas en este estudio están dentro de las dadas para mujeres no embarazadas.

Puesto que no se conocen tablas para determinar la superficie corporal de las mujeres embarazadas, no hemos hecho tentativa alguna de correlacionar la Capacidad Vital con la superficie de la paciente. La estimación de la normal para una paciente dada es, talvez, hecha de un modo más satisfactorio por comparación entre los niveles pre-partum y la última lectura post-partum. Esta última lectura se supone ser la Capacidad Vital normal para tal paciente.

La Capacidad Vital no se encontró que disminuyera durante los últimos tres meses de embarazo. Las observaciones pre-natales son las mismas o ligeramente más altas que la última lectura post-partum.

Contrariamente a los resultados de otros autores (5) no hubo reducción 
durante el último mes lunar del embarazo. Las cifras son las mismas o ligeramente más altas que las de las semanas anteriores.

Es más, de las 33 pacientes con observaciones pre y post-partum sólo 7 mostraron después del parto, un aumento de 100 centímetros cúbicos o más por encima de la última cifra pre-partum.

Inmediatamente después del parto hay una caída indisputable de la capacidad vital. Nuestras observaciones durante el primer día postpartum fueron las más bajas de todas las anotadas. Varias sugerencias han sido hechas para explicar este cambio: repentina disminución del perimetro torácico, cambios de tono en los músculos de la respiración o talvez la fatiga general que sigue al parto.

La Capacidad Vital aumenta firmemente-durante la primera semana post-partum para alcanzar casi su nivel normal al séptimo día.

En la posición sentada, la menor y la mayor de las determinaciones pre-natales fueron: 1.900 centímetros cúbicos y 4.200 centímetros cúbicos respectivamente. La menor y la mayor post-natal fueron: 1.450 y 3.900 centímetros cúbicos.

La determinación rutinaria de la capacidad vital es, pues, un test responsable para descubrir y estimar la descompensación cardíaca. Cualquier disminución notoria, excepto el primer dia post-partum, es evidencia de descompensaciór

\section{Velocidad de circulación.}

Uno de los factores importantes en el mantenimiento de la eficiencia circulatoria es la velocidad de la masa sanguínea.
Mucho ha sido escrito durante los últimos años acerca de la determinación del tiempo de circulación y su valor como medio diagnóstico en las enfermedades cardio-vasculares.

Por largo tiempo se ha aceptado que la velocidad de la circulación está aumentada durante el embarazo y ésta es una de las razones más frecuentemente aducidas para explicar la frecuencia de soplos funcionales durante esta época. Sin embargo es tan sólo en años recientes que en realidad se han hecho determinaciones de la velocidad de la corriente sanguínea durante el embarazo. De nuevo aquí, también existe gran desacuerdo en los resultados de los diferentes autores.

Tiempos de Circulación Brazo a Lengua y Brazo a Pulmón, fueron hechas al mismo grupo de pacientes embarazadas en el que bajo condiciones correspondientes, determinaciones de Capacidad Vital se habian practicado.

Se usó éter diluído en el Tiempo de Circulación Brazo a Pulmón, ya que Hussey (41) ha demostrado que. el éter es el agente más responsable y que da cifras más exactas.

Se usó solución de Sacarina al $50 \%$ en agua destilada, para la determinación de Tiempo de Circulación Brazo a Lengua.

El fundamento de los tests y sus técnicas han sido tan ampliamente publicados y comentados en libros y revistas recientes, que no nos detendremos en describirles.

Un total de 356 determinaciones fueron hechas, incluyendo en ellas 187 Tiempos de Circulación Brazo a Lengua y 169 Brazo a Pulmón. 
En veinticuatro pacientes hubo observaciones pre-natales solamente. Treinta y una tuvieron determinaciones pre y post-natales. El numero de determinaciones pre-natales varić entre dos y seis por paciente y fueron hechas durante los últimos tres meses del embarazo. La mayoría de las observaciones post-natales fueron hechas el primer día, el séptimo día y cinco a seis semanas después del parto.

De las 55 pacientes originales, varias fueron eliminadas por las siguientes causas: una rehusó el test; cuatro mostraron muy poca cooperación y en cinco las venas del antebrazo no eran adecuadas.

\section{Resultados.}

Se encontraron variaciones considerables en los resultados de la Velocidad de Circulación. Aunque los valores obtenidos son ligeramente más altos que las cifras normales, en el mayor número de los casos estaban dentro de los límites dados para mujeres no embarazadas.

En las determinaciones del Tiempo de Circulación Brazo a Lengua, la menor y la mayor cifra pre-natal fue: 7 segundos y 25 segundos respectivamente; la menor y la mayor post-partum fue 8 y 20 segundos respectivamente.

En las determinaciones del Tiempo de Circulación Brazo a Pulmón, la menor y la mayor cifra pre-natal fue: 4 y 15 segundos respectivamente; la menor y la mayor cifra postpartum fue: 5 segundos y 14,5 segundos respectivamente.

Con el objeto de presentar todos los resultados en conjunto se dibu- jaron las gráficas I y II y en ellas se han trazado los valores promedios en una línea continua.

El único resultado notor io constante es una ligera disminución del Tiempo de Circulación en el primer día post-partum, comparada con la última cifra pre-partum, tanto de la Circulación Brazo a Lengua como Brazo a Pulmón. Por lo demás las variaciones en los resultados son tan considerables, que no creemos pueda sacarse deducción alguna acerca de si la velocidad de circulación está aumentada durante el embarazo o no. Más aún y por la misma razón, no puede darse un modelo prefijado de las variaciones del Tiempo de Circulación durante los últimos meses del embarazo.

La explicación del por qué tan amplia variación en los resultad.os estriba, creo en varias causas como:

La labilidad de la circulación durante el embarazo. Probst (58) entre otros, ha señalado que la circulación de la mujer embarazada, auncuando bien balanceada, presenta una marcada sensibilidad a sobrecargas experimentales.

En la realización de los tests de Velocidad de Circulación, debe usarse la punción venosa, y punción venosa significa agujas y dolor. La mayoría de nuestras pacientes a pesar de reposo y condiciones basales, se tornaban nerviosas y muy aprehensivas con la mera perspectiva del test. Decían: "me asustan las agujas". Una de ellas se desmayó simplemente a la vista de la aguja. No es raro que con el éter se presenten ligeras reacciones dolorosas a lo largo del brazo y esto ha sido descrito por otros autores también. Así, pues. 


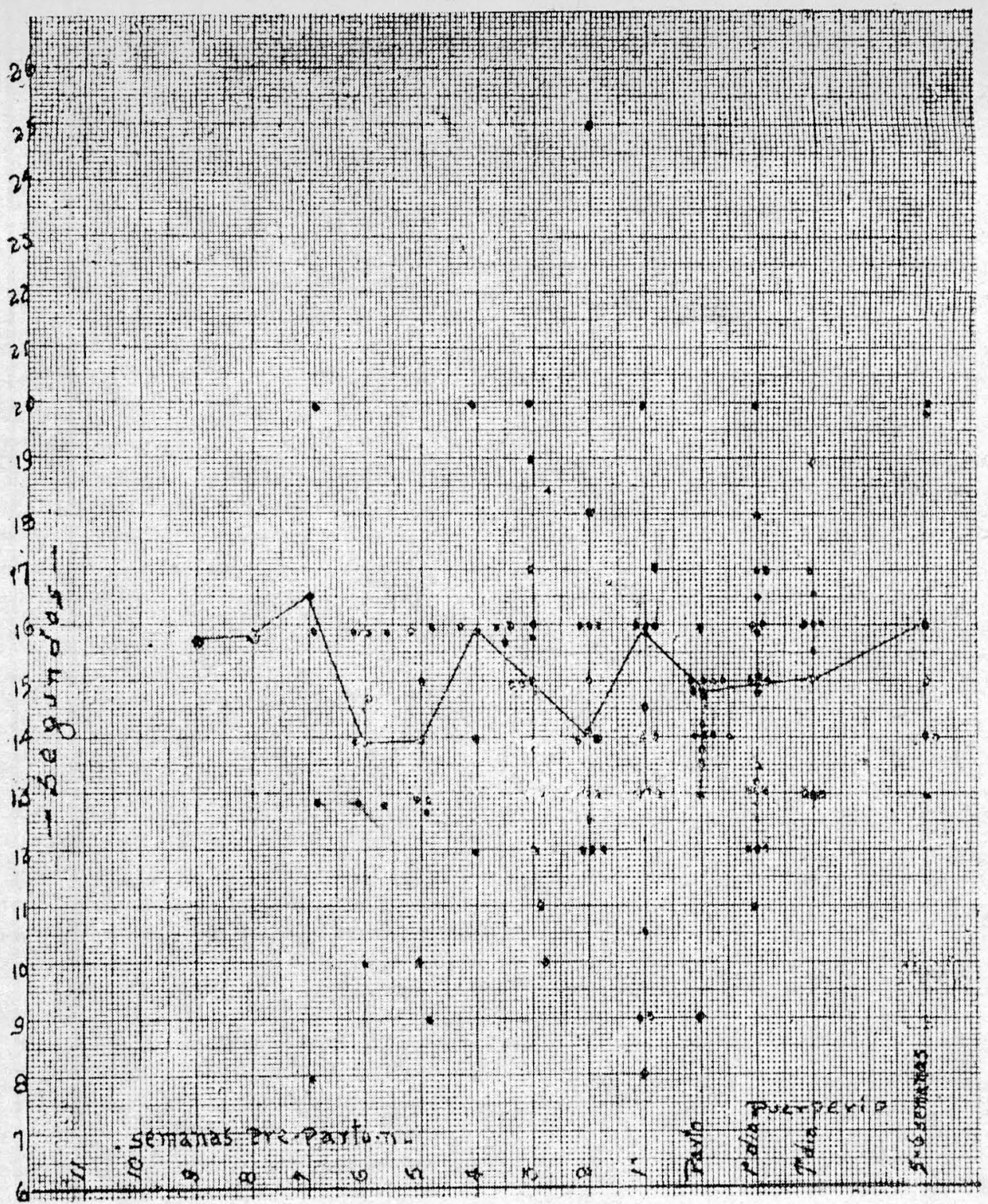

\section{GRAFICA I}

\section{TIEMPOS DE CIRCULACION BRAZO a LENGUa" DURANTE EL PERIODO PRE Y POST-PARTUM}

Los pantos negros representan observaciones individuales, la línea continua los resultados promedios. Unicamente las pacientes con observaciones pre y post-partum fueron incluídas en la gráfica. El tiempo de circulación "brazo a lengua" fue hecho con Solución de Sacarina al $50 \%$. 


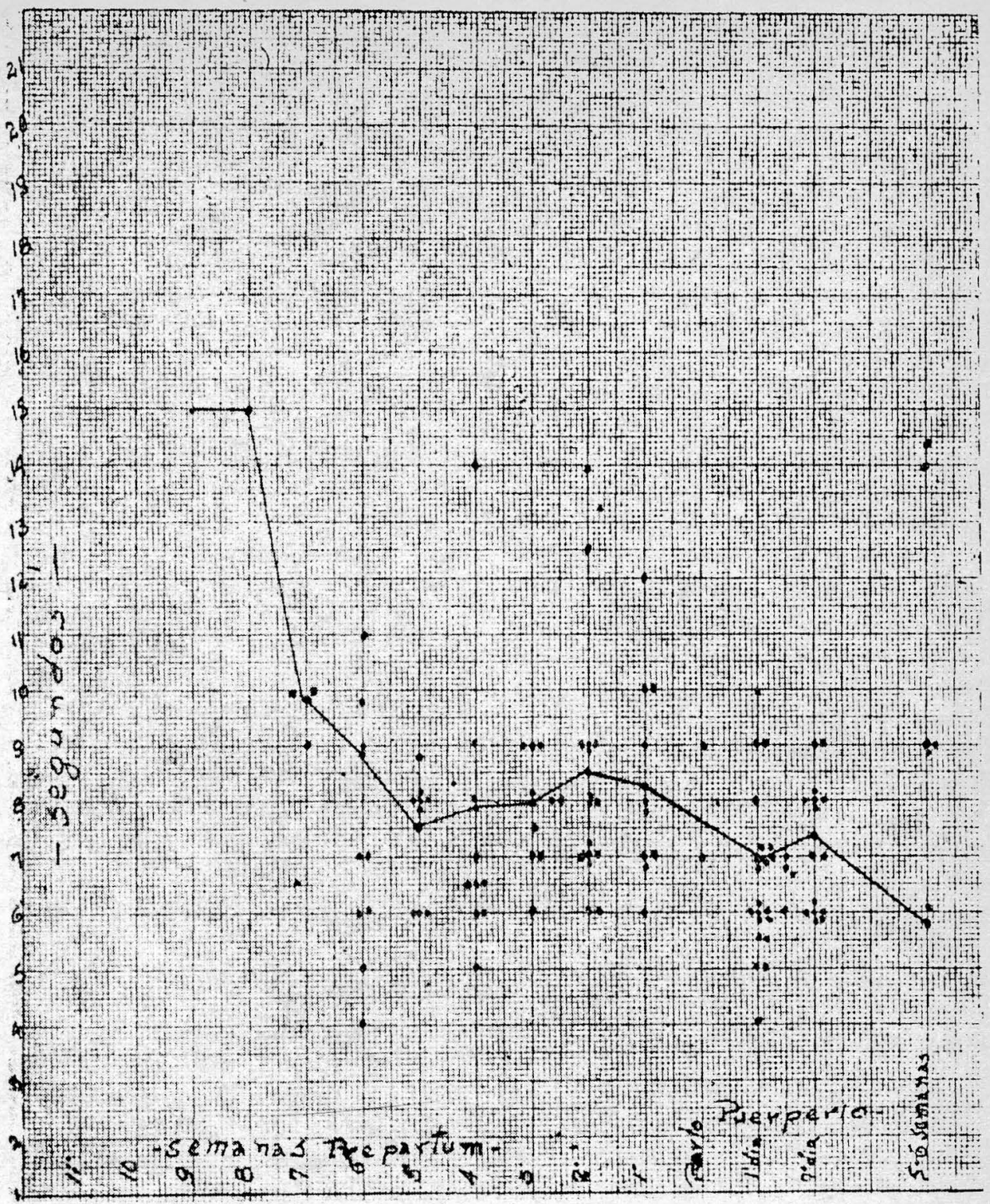

\section{GRAFICA II}

\section{TIEMPOS DE CIRCULACION "BRAZO A PULMON" DURANTE EL PERIODO PRE Y POSTPARTUM}

Los puntos negros representan observaciones individuales, la línea continúa los resultados promedios. Unicamente las pacientes con observaciones pre y post partum fueron incluíudas en la Gráfica. El tiempo de circulación "brazo a pulmón" fue hecho con éter diluido. 
dolor, nerviosidad, aprehensión, etc., darán variaciones de la frecuencia del pulso y del débito cardíaco, las que naturalmente se reflejarán en otros factores de la mecánica de la circulación.

Es más, con excepción del caso en que el operador percibe el olor a éter, la determinación del Tiempo de Circulación es un test puramente subjetivo y por lo tanto bajo el dominio de influencias emocionales. Una de nuestras pacientes percibió un sabor amargo en vez del sabor dulce peculiar a la Sacarina. Muchas de ellas notaron el sabor de la Sacarina al pinchar la vena y antes de que la solución hubiera sido inyectada, esto sucedió más a menudo cuando la enferma ya conocía el test.

Tan sólo otros cuatro autores han presentado una serie tan numerosa de velocidades de circulación durante el embarazo, a saber: Cohen y Thompson (15) y Bernstein y Simkins (8). Al ojear las gráficas de ellos, similares a las presentadas, nos sorprendimos al notar que los puntos negros, que representan observaciones individuales, estaban tan desparramados como los nuestros, aunque ellos también presentaban una curva de valores promedios.

Esser (26) en determinaciones de Tiempo de Circulación Brazo a Lengua con Sacarina, en mujeres normales no embarazadas, efectuadas dos veces a cada una en las mismas condiciones, comprobó que: "es regla encontrar diferencias considerables entre los resultados duplicados".

Entiéndase bien que no pretendemos negar el valor de los resultados del Tiempo de Circulación como un test "a grosso" de descompensación cardíaca en disturbios cardio-vasculares. Las determinaciones hechas en pacientes descompensadas, mostraron muy claramente las dificultades circulatorias puesto que varias veces se hallaron valores $\tan$ altos como 40 segundos.

Sin embargo, con excepción de la disminución del tiempo de circulación en el inmediato post-partum, de nuestra amplia escala de resultados, no pueden derivarse conclusiones respecto a las variaciones dentro de límites normales de la Velocidad de Circulación durante el embarazo.

Nota: Las observaciones anteriores sobre Capacidad Vital y Tiempo de Circulación fueron hechas, como dijimos, en mujeres normales embarazadas. Se hicieron también determinaciones de Capacidad Vital, Presión Venosa y Tiempo de Circulación en muchas pacientes cardíacas pero como este estudio no fue sistematizado, los resultados no se presentan.

\section{Sumario.}

Impresionado por las muchas controversias existentes acerca de las enfermedades cardíacas y la mecánica circulatoria durante el embarazo, fue hecho este trabajo.

Se encontró una incidencia de enfermedad orgánica cardíaca de $0,41 \%$ en 16.385 partos en el Desloge y el St. Mary's Hospitals del Grupo de la Universidad de St. Louis, durante los ocho años, de 1939 a 1946 inclusive. El Reumatismo Cardíaco fue la causa en el $85,3 \%$ del grupo de pacientes mencionados El resto de las lesiones fueron debi. 
das a Enfermedad Cardíaca Congénita, Hipertensiva, Cardio-vasculorenal y Taquicardia paroxística.

Se hicieron consideraciones generales sobre las cardiacas durante el embarazo y al mismo tiempo se presentó un repaso de la literatura al respecto. La moderna y feliz tendencia hacia un conservatismo en el manejo de la embarazada cardíaca fue señalada y se dieron sugestiones adicionales.

Se determinó la duración del trabajo y el tamaño promedio de los recién nacidos de madres cardiacas, basados en los records de 76 pacientes de los hospitales Desloge, St. Mary's, St. Anthony's St. John's, y DePaul del Grupo de la Universidad de St. Louis.

Se hizo un total de 1260 determinaciones de Capacidad Vital en un grupo de 55 mujeres blancas normales de la Clínica Obstétrica del Desloge Hospital.

Sobre el mismo grupo de pacientes se hizo un total de 356 observaciones de Tiempos de Circulación, que incluyen 187 determinaciones Brazo a Lengua y 169 Braza a Pulmón.

Los resultados son presentados $\mathrm{y}$ comentados.

\section{Conclusiones.}

1) El tratamiento de las enfermeciades cardíacas durante el embarazo al presente es más conservador de lo que ha sido en el pasado. A una disminución de la mortalidad materna, se ha llegado mediante rígida vigilancia y control periódico estricto de la paciente cardíaca embarazada. La importancia del descubrimiento precoz de la descompen- sación, el reposo en cama y otras medidas han sido señaladas.

2) La inducción médica del trabajo y el parto forzado no son medidas seguras en la mujer cardíaca y deben ser usadas muy cautelosamente $\mathrm{y}$ por ningún motivo mientras la paciente esté en descompensación congestiva aguda.

3) Las pacientes cardiacas no tienen partos más cortos como se cree. El promedio de duración de trabajo encontrado fue de 14 horas $91 / 2 \mathrm{mi}-$ nutos para primíparas y 7 horas 33 minutos para multíparas. Comparadas estas cifras con los promedios normales parecen un tánto cortas. Esta disparidad es fácilmente comprensible si se recuerda que la aplicación de forceps en la cardíaca reemplazó usualmente el segundo período del trabajo.

4) No hubo diferencia entre el tamaño de los recién nacidos de madres normales y los nacidos de madres cardíacas. El tamaño promedio de aquellos nacidos de cardíacas fue: peso: 3135,5 gramos. Longitud: 50,1 centímetros.

5) No se encontró disminución de la capacidad vital durante los últimos meses del embarazo. Las cifras pre-natales son las mismas o ligeramente más altas que la última lectura post-partum. Al contrario de lo hallado por otros investigadores tampoco se notó reducción alguna durante el último mes lunar del embarazo. Hubo sí una indiscutible caída de la capacidad vital inmediatamente después del parto. Las determinaciones de capacidad vital son un test responsable para el descubrimiento y estimación de la descompensación cardíaca durante el embarazo. 
6) Fueron halladas variaciones considerables en los resultados de las determinaciones de la Velocidad de Circulación durante el embarazo. Aunque los valores obtenidos son ligeramente más altos que los dados para mujeres no embarazadas, en la mayor parte de los casos las cifras estaban dentro de los límites normales. Pueden verse por nuestra amplia escala de resultados que con excepción de una disminución del Tiempo de Circulación en el inmediato post-partum, no puede derivarse conclusión alguna de las variaciones dentro de los límites normales de las determinaciones de la velocidad de circulación durante el embarazo.

\section{BIBLIOGRAFIA}

\section{Libros:}

(1) Hamilton B. E. y Thomson J. $K$., The Heart in Pregnancy and the Childbearing Age. Little Brown and Company, Boston, 1941.

(2) Jensen J., The Heart in Pregnancy C. V. Mosby and Con pany. St. Louis, 1938.

(3) Stander H. J., Textbook of Obstetrics. D. Appleton-Century. Company. New York, 1945.

(4) Grollmann A., The Cardiac Output of Man in Health and Disease. Charles C. Thomas, Editor, Baltimore, 1932.

\section{Journals:}

(5) Alward H. C., Observations on the Vital Capacity During the Last Month of Pregnancy and Puerperium. American Journal of Obstetrics and Gynecology, 20-373-381, 1930.

(6) Baer S., The Clinical Application of the Determination of the
Circulation Time. Annals of Internal Medicine, 13-2246-2251, 1940.

(7) Bassu M. N., Heart Disease in Pregnancy, Calcutta Medical Journal, 36-30-37, 1939.

(8) Bernstein M., and Simkins S., On the Magnesium Sulphate and Ether Circulation Times During Pregnancy. American Journal of Obstetrics and Gynecology, 41-970978, 1941.

(9) Boyer N. H., and Nadas A. S., The Ultimate Effect of Pregnancy on Rheumatic Heart Disease. Annals of Internal Medicine, 20-99-107, 1944.

(10) Burwell C. S., The Placenta as a Modified Arterio-Venous Fistula, Considered in Relation to the Circulatory Adjustments to Pregnancy. American Journal of Medical Science, 195-1-7, 1938.

(11) Burwell C. S., et al., Circulation During Pregnancy. Archives of Internal Medicine, 62-979-1003, 1938.

(12) Capelläri M., Estudio Gráfico de los Ruidos Cardíacos en el Foco Pulmonar en Cincuenta Embarazadas Normales. Revista Argentina de Cardiología, 5-155-160, 1938.

(13) Carr. F. B., y Hamilton B. E., Five Hundred Women with Serious Heart Disease Followed Through Pregnancy and Delivery, American Journal of Obstetrics and Gynecology, 26-824-839, 1933.

(14) Carr F. B., Heart Disease in Pregnancy. New England Journal of Medicine, 219-231-232, 1938.

(15) Cohen M. E., y Thomson J. K., The Volocity of Blood Flow and Related Aspects of the Circulation in Normal Pregnant Women. Journal of Clinical Investigation, 15-607-625, 1936. 
(16) Cohen M. E., y Thomson J. K., Studies on tre Circulation in Pregnancy. Journal of the American Medical Association, 112-1556-1562, 1939.

(17) Corwin J., y Henrick W. W., y Valentine M., y Wilson J. J., Pregnancy and Heart Disease. American Journal of Obstetrics and Gynecology, 13-617-622, 1927.

(18) Clahr J., y Greenstein N. M., y Klein M. D., Circulation Time Studies in Pregnant Women with Rheumatic Heart Disease. American Journal of Obstetrics and Gynecology, 38-39-47, 1939.

(19) Clahr J., y Klein M. D., y Greenstein N. M., Rheumatic Heart Disease in Pregnant Women. New York State Journal of Medicine, 401272-1247, 1940.

(20) Daichman I., y Kernefeld G., Pregnancy complicating Heart Disease. American Journal of Obstetrics and Gynecology, 30-386-391, 1935.

(21) Daly P. A., The Heart in Pregnancy. Journal of the American Medical Association, 82-1439-1441, 1924.

(22) Danforth W: C., The Management of Pregnancy and Labor in the Presence of Heart Disease. Illinois Medical Journal, 74-88-91, 1938.

(23) Easby M. H. The Early Recognition of Cardiac Insufficiency in Pregnancy. Medical Clinics of North America, 21-1059-1971, 1937.

(24) Eastman N. J., Heart Disease in Pregnancy: The Respective Duties of Internist and Obstetrician. Medical Clinics of North America, 21-1407-1416, 1937.

(25) Embring G. y Sulton D. C., Heart Disease in Pregnancy. Illinois Medical Journal, 72-147-153, 1937.
(26) Esser K. H., ỳ Berliner K., Duplicate Measurements of CircuCirculation Time made with the Saccharin Method. Annals of Internal Medicine, 19-64-69, 1943.

(27) Flaxman N., Pregnancy and Heart Disease. American Journal of Obstetrics and Gynecology. 39-814817, 1940.

(28) Fordel, citado por Stander (3) pagina 290.

(29) Frankenthal L. E., Pregnancy in the Cardiac. Surgical Clinics of North America, 23-35-43, 1943.

(30) Fraser, Maternal Mortality and Morbidity. Journal of the American Medical Association, 105-14811486, 1935.

(31) Gammeltfolt S. A., Recherches sur la Debité Cardiaque par Minute Pendant la Grossesse. Comptes Rendus de la Societé de Biologie, 94-1099-1101, 1926.

(32) Gammeltfolt S. A., The Heart in Pregnancy. Surgery, Gynecology and Obstetrics, 46-382-390, 1928.

(33) González S. L., Cardiopatías y Embarazo. "El Día Médico", 15-11941196, 1943.

(34) Gorembergh H. y McGleary J., Rheumatic Heart Disease in Pregnancy. American Journal of Obstetrics and Gynecology, 41-44-52, 1941.

(35) Gorembergh H., Rheumatic Heart Disease: A Controllable Complication in Pregnancy. American Journal of Obstetrics and Gynecology, 45-835-840, 1943.

(36) Hamilton B. E., Sixteen years of Experience with Heart Disease in Pregnant Women. American Heart Journal, 14-555-561, 1937.

(37) Hamilton B. E., Heart Disease in Pregnancy. The Journal of the Missouri Medical Association, 44-1721, 1947. 
(38) Hermmann G. y King E. L., Cardio-vascular Disturbances in the Obstetrics Patients with Special Reference to Electrocardiographic $\mathrm{Ob}$ servations. Journal of the American Medical Association, 95-1472-1476, 1930.

(39) Hoffmann G. L., y Jeffers W. J., Rheumatic Heart Disease complicating Pregnancy: Study of Sixty-One Fatalities. American Journal of Medical Science, 204-157-163, 1942.

(40) Hollander A. G., y Goldsmith J. W., Acute Rheumatic Fever Complicating Advanced Pregnancy. American Journal of Obstetrics and Gynecology, 42-335-343, 1941.

(41) Hussey H. H., y Kats S., The Comparative Valus of the Ether and Paraldehyde as Agents for Measurement of the Arm to Lung Circulation Time in Fifty Patientes with, and Fifty Patientes without Heart Failure. American Journal of Medical Science, 201-669-676, 1941.

(42) Hussey H. H., y Wallace J. J., y Sullivan J. C., The Value of the Combined Measurements of the Venous Pressure and Arm to Tongue and Arm to Lung Circulation Time in the Study of Hearth Failure. American Heart Journal, 23-22-36, 1942.

(43) Jensen J., y Wegner C., y Keys B., y Smith H., Heart Disease in Pregnancy. American Journal of Obstetrics and Gynecology, 39-443$449,1940$.

(44) Lamb A. E., Heart Disease in Pregnancy. American Journal of Obstetrics and Gynecology. 34-456469, 1937.

(45) Mackenzie, citado por Tilghmann.(71).
(46) McLennan C. E., Antecubital and Femoral Venous Pressure in Normal and Toxemic Pregnancy. American Journal of Obstetrics and Gynecology, 45-568-590, 1943.

(47) Mendelson C. L., y Pardee H. E., Congenital Heart Disease During Pregnancy. American Journal of Medical Science, 202-392-402, 1941.

(48) Mendelson C. L., Pregnancy and Coarctation of the Aorta. American Journal of Obstetrics and Gynecology, 39-1014-1021, 1940.

(49) Mendelson C L., The Managemente of Delivery in Pregnancy Complicated by Serious Heart Disease. American Journal of Obstetrics and Gynecology, 48-329-338, 1944.

(50) Mendelson C. L., y Pardee H. E., The Pulse and Respiration Rates During Labor as a Guide to the Onset of Cardiac Failure in Women with Rheumatic Heart Disease. American Journal of Obstetrics and Gyncology, 44-370-383, 1942.

(51) Mitchell F. V., y Davis S. F., y Hollander A. G., Complete Heart Block Complicating Pregnancy. American Journal of Obstetrics and Gynecology, 45-340-343, 1943.

(52) Muser J. H., y Sodeman W. A., Heart Changes of Unusual Etio$\log y$ in Pregnancy. Transactions of the Association of American Physicians, 54-181-187, 1939.

(53) Oppel T. W., Congestive Heart Failure in Pregnancy. American Journal of Obstetrics and Gynecology, 39-24-32, 1940.

(54) Osler, citado por Embring y Sulton (25).

(55) Pardee H. E., The fitness for Fregnancy of Patients with Heart Disease. Journal of American Medical Association, 78-1188-1890, 1922. 
(56) Pardee H. E. Experiences in the Management of Pregnancy complicated by Heart Disease. American Journal of Obstetrics and Gynecology. 17-255-262, 1929.

(57) Peters M., Accidents that may Happen to Pregnant Women Suffering From Diseases of the Heart. British Medical Journal, 2-289-290, 1874.

(58) Probst B., Functionsprünfüng des Kreislanfes fesunder Schwangerer. Archives für Gynakologie, 166-59-62, 1938.

(59) Reid W D., The Heart in Pregnancy. Journal of the American Medical Association, 95-1468, 1930.

(60) Sampson J. J., y Rose E. M., y Quinn R., Estimation of the work in Obstetrical Labor and its Significance in Heart Disease. American Journal of Obstetrics and Gynecology, 49-719-734, 1945.

(61) Semisch C. W., The Management of the Pregnant Cardiac Patient. Medical Clinics of North America, 29-1449-1453, 1945.

(62) Sheehan H. L., y Sutherland A. M., The Pathology of Heart Disease in Pregnancy. The Journal of Obstetrics and Gynecology of the British Empire, 47-597-668, 1940.

(63) Sodeman W. A., y Burch G. E., The precipitating Causes of Congestive Heart Failure. American Heart Journal, 15-22-27, 1938.

(64) Sodeman W. A., Cardiac Changes in Pregnancy Unrelated to the Usual Etiological Type of Heart Disease. American Heart Journal, 19385-397, 1940.

(65) Sodeman W. A., y King E. L., The Heart in pregnancy: Prognosis Aspects. Southern Medical Journal, 37-235-239, 1944.
(66) Stander H. J., y Duncan E. E., y Sisson W. E., Heart Output During Pregnancy. American Jornal of obstetrics and Gynecology, 11-44-50, 1926.

(67) Stander H. J., y Cadden J. F., The Cardiac Output in Pregnant Women. American Journal of Obstetrics and Gynecology, 24-13-19, 1932.

(68) Stander H. J., Treatment of Heart Disease in Pregnancy. American Journal of Obstetrics and Gynecology, 44-714-722, 1942.

(69) Thomson J. K., y Cohen M. E., Vital Capacity Observations in Normal Pregnant Women. Surgery, Gynecology and Obstetrics, 66-591-603, 1938 .

(70) Thomson J. K., y Reid W., y Cohen M. E., Venous Pressure in Normal Pregnant Women with Compensated and Decompensated Heart Disease and in Toxemia. American Journal of Medical Science, 198-665679, 1939.

(71) Tilghmann R. C., Heart Disease in Pregnancy. New York State Journal of Medicine, 39-248-253, 1939.

(72) Turino T. R., y Antony A. T., Heart Disease in Pregnancy: Obstetricial Aspects. American Journal of Surgery, 41-453-457, 1938.

(73) Turino T. R., y Wallace J. T., Resume of Cardiac Disease in Pregnancy for Five Year Period. American Journal of Obsterics and Gynecology, 45-526-529, 1943.

(74) Weyler H., y Dustin C., Paroxisma Tachycardia in Pregnancy New England Journal of Medicine, 227-785-787, 1942.

(75) White, Marx citado de Jensen (2), página 250 .

(76) Wirth R. W., Heart Disease in Pregnancy. Mississippi Doctor. 15$1-5,1937$ 